\title{
Groupes de Lie compacts de transformations de l'espace euclidien et les sphères comme espaces homogènes
}

\section{Doctoral Thesis}

Author(s):

Poncet, Jean

Publication date:

1959

Permanent link:

https://doi.org/10.3929/ethz-a-000103804

Rights / license:

$\underline{\text { In Copyright - Non-Commercial Use Permitted }}$ 
Prom. No. 2784

\title{
GROUPES DE LIE COMPACTS DE TRANSFORMATIONS DE L'ESPACE EUCLIDIEN ET LES SPHÈRES COMME ESPACES HOMOGÈNES
}

\author{
THÈSE \\ PRESENTEE
}

A L'ÉCOLE POLYTECHNIQUE FÉDÉRALE, ZURICH

POUR L'OBTENTION DU GRADE DE

DOCTEUR ES SCIENCES MATHÉMATIQUES

PAR

\section{JEAN PONCET}

DE LIGNEROLIE (Vaud) ET NEYRUZ (Fribourg)

Rapporteur: Prof. Dr. A. Borel

Corapporteur : Prof. Dr. B. EckMaNN

ORELC FUUSSI ARTS GRAPHIQUES S.A., ZURICH 


\title{
Groupes de LIE compacts de transformations de l'espace euclidien et les sphères comme espaces homogènes
}

\author{
par JEAN PONCET, Zurich
}

\section{Introduction}

C'est un problème ouvert de savoir ce qui caractérise topologiquement les groupes de transformations linéaires parmi les groupes de LIE compacts de transformations de l'espace euclidien $E_{n}$. Les résultats les plus complets dans ce sens, pour les petites dimensions, ont été énoncés par MonTGomery et ZIPPIN et prennent une signification particulière pour les fondements de la géométrie en termes de groupes de congruences (voir [7], chap. VI): un groupe compact opérant sur $E_{n}, n \leqq 3$, - on démontre d'abord qu'il est de LIE est équivalent topologiquement à un groupe orthogonal si l'on suppose seulement qu'il est connexe. Si $G$ n'est pas connexe, il n'opère pas nécessairement linéairement sur $E_{3}$, comme le montre un exemple de BING [1] d'une transformation involutive de $E_{3}$ qui possède des points fixes et qui n'est différentiable, à plus forte raison linéaire, pour aucune structure différentiable de $E_{3}$. Si $n>3$, la connexion de $G$ ne suffit plus pour qu'il soit équivalent à un groupe compact de LIE de transformations différentiables de $E_{n}$ : [8] donne un exemple de groupe isomorphe à $S O(2)$, le groupe des rotations de $E_{2}$, qui opère sur $E_{4}$ et qui n'est différentiable pour aucune structure différentiable de $E_{4}$. Il s'ensuit que pour $n>3$ d'autres conditions topologiques que la connexion et la compacité de $G$ seront nécessaires pour qu'il soit linéaire sur $E_{n}$. Dans ce qui suit, nous montrons que $G$ est linéaire sur $E_{n}$ si $G$ est compact et connexe et s'il existe au moins une orbite de dimension $n-1$, ou, sous les mêmes hypothèses pour $G$, si les orbites de $E_{n}$ sont des sphères $S_{n-2}$ et des points fixes. Nous utiliserons pour cela les résultats de [7] et de [9]. De plus $G$ opère aussi linéairement s'il est isomorphe à $S O(n-1)$ et s'il existe des orbites de dimension $n-2$.

Dans [7], MonTgomery et ZrPPIN étudient les groupes compacts et connexes de transformations de $E_{n}$ sous l'hypothèse qu'il existe une orbite de dimension $n-1$. Ils montrent que toutes les orbites sauf un point fixe sont de dimension $n-1$, qu'elles possèdent le même groupe d'isotropie et sont donc homéomorphes entre elles. En outre, les orbites de dimension $n-1$ ont même homologie entière qu'une sphère $S_{n-1}$. Comme une orbite $(n-1)$ dimensionnelle d'un espace $n$-dimensionnel localement euclidien est locale- 
ment connexe ([7], chap. VI, corollaire de la p. 248) et qu'un groupe compact et effectif de transformations d'un tel espace est de LIE si ses orbites sont localement connexes ([7], chap. VI, Th. 1, p. 244), $G$ est de LIE. La structure de $E_{n}$ comme espace de transformations de $G$ est assez simple puisque le complémentaire du point fixe est un espace fibré trivialement en orbites, d'où l'existence d'un rayon qui est une section pour toutes les orbites. Tout ceci fait conjecturer que $G$ opère linéairement sur $E_{n}$, ce que nous démontrerons en utilisant un théorème de $\mathrm{A}$. Bores ([2] et non publié; voir aussi [3]), d'après lequel les orbites de dimension $n-1$, ayant même homologie entière que des sphères $S_{n-1}$ et étant simplement connexes pour $n-1>1$ (pour $n-1=1$ il n'y a rien à démontrer), sont nécessairement des sphères $S_{n-1}$. Ceci pourrait être établi sans considérations d'homologie si l'on supposait que $G$ opère différentiablement. En effet, d'après le théorème de BochNer ([7], chap. V), $G$ agit alors linéairement dans un voisinage invariant du point fixe; les orbites de ce voisinage sont sur des sphères $S_{n-1}$ et doivent se confondre avec ces sphères si elles sont de dimension $n-1$. Cela est vrai globalement puisque les orbites différentes du point fixe sont des espaces homogènes isomorphes.

Dans [9] Montgomery, Samelson, Yang montrent que $G$ compact, connexe, de LIE, est équivalent, comme groupe de transformations de $E_{n}$, à un groupe linéaire s'il existe des orbites de dimension $n-2$ et si les transformations de $G$ sont différentiables une fois sur $E_{n}$. Une partie de leur raisonnement n'utilise pas l'hypothèse de différentiabilité et montre que les orbites de dimension $n-2$ ont même groupe d'isotropie, leur réunion $R$ est un ouvert dense dans $E_{n}$, l'espace quotient $E_{n} / G$ est homéomorphe à une cellule à deux dimensions diminuée d'un point frontière, l'intérieur homéomorphe à $E_{2}$ et la frontière homéomorphe à $E_{1}$ étant respectivement $R / G$ et $S / G, S$ désignant le complémentaire de $R$ dans $E_{n}$. Ceci nous permet de démontrer sans hypothèses de différentiabilité que $G$ opère linéairement si ses orbites sont des sphères $S_{n-2}$ et des points fixes, et aussi, sans hypothèses sur les orbites de dimension $n-2$, si $G$ est isomorphe à $S O(n-1)$.

Il n'est pas impossible que seule l'existence d'orbites $S_{n-2}$ soit suffisante pour que $G$ soit linéaire; nous comptons reprendre la question ailleurs. Toutefois nous montrons par des considérations d'homologie que le complémentaire $S$ de la réunion des orbites $S_{n-2}$ se réduit à des points fixes s'il ne possède qu'un nombre fini de "singularités».

Le théorème cité de Montgomery et ZiPPIN sur les groupes compacts et connexes qui opèrent sur $E_{n}, n \leqq 3$, est une conséquence immédiate des deux premiers résultats de ce travail.

Je remercie ici Monsieur A. BoreL pour les conseils qu'il m'a donnés. 